\title{
A Local Mechanism by which Alcohol Consumption Causes Cancer
}

\author{
Miguel López-Lázaro \\ Department of Pharmacology, Faculty of Pharmacy, University of Seville, C/ Profesor Garcia \\ Gonzalez 2, 41012 Sevilla, Spain; mlopezlazaro@us.es; Tel: +34 9545563 48; Fax: + 34954556074.
}

\begin{abstract}
Epidemiological data indicate that $5.8 \%$ of cancer deaths world-wide are attributable to alcohol consumption. The risk of cancer is higher in tissues in closest contact on ingestion of alcohol, such as the oral cavity, pharynx and esophagus. The risk of these cancers is increased even in people who have only one alcoholic drink per day. However, since ethanol is not mutagenic and the carcinogenic metabolite of ethanol (acetaldehyde) is mostly produced in the liver, it is not clear why alcohol use preferentially exerts a local carcinogenic effect. It is well known that ethanol causes cell death at the concentrations present in alcoholic beverages; however, this effect has been overlooked probably because dead cells cannot give rise to cancer. Here I discuss that the cytotoxic effect of ethanol on the cells lining the oral cavity, pharynx and esophagus activates the division of the stem cells located in deeper layers of the mucosa to replace the dead cells. Every time stem cells divide, they become exposed to unavoidable errors associated with cell division (e.g., mutations arising during DNA replication and chromosomal alterations occurring during mitosis) and also become highly vulnerable to the genotoxic activity of endogenous and exogenous DNA-damaging agents (e.g., reactive oxygen species, acetaldehyde and tobacco carcinogens). Alcohol consumption probably increases the risk of developing cancer of the oral cavity, pharynx and esophagus by promoting the accumulation of cell divisions in the stem cells that maintain these tissues in homeostasis. Because the cytotoxic activity of ethanol is concentration-dependent, the risk of these cancers will not only increase with increasing amounts of ethanol, but also with increasing concentrations; an ounce of whisky is probably more carcinogenic when taken undiluted than when taken mixed with non-alcoholic beverages. The local cytotoxic effect of ethanol can also explain the known synergistic effect of alcohol and tobacco use on the risk of these cancers. Understanding the mechanisms of carcinogenicity of alcohol is important to reinforce the epidemiological evidence and to raise public awareness of the strong link between alcohol consumption and cancer.
\end{abstract}

Keywords: alcohol; cancer; ethanol; tobacco; carcinogenesis; oral cavity; pharynx; esophagus; stem cells; stem cell division theory of cancer

\section{Introduction}

Epidemiological evidence strongly suggests that moderate to heavy alcohol consumption increases the risk of cancer of the oral cavity, pharynx, larynx, esophagus, liver, colon, rectum and female breast [1-3]. Recent estimates indicate that the relative risk for heavy drinkers compared with nondrinkers and occasional drinkers is 5.13 for oral and pharyngeal cancer, 4.95 for esophageal 
cancer, 2.65 for laryngeal cancer, 2.07 for liver cancer, 1.44 for colorectal cancer and 1.61 for breast cancer [3]. Light alcohol drinking (up to one drink per day) increases the risk of cancer of the oral cavity, pharynx, esophagus and breast, but not of the liver, larynx, colon and rectum [4]. It has been estimated that $5.8 \%$ of cancer deaths world-wide are attributable to alcohol use [5]. However, the biological mechanism by which alcohol causes cancer is unclear $[1,2,6,7]$; this is often raised as an objection to accept the strong epidemiological link between alcohol consumption and cancer [2].

Cancer is caused by exposure to mutagenic agents, but ethanol is not mutagenic. Most of the ingested ethanol is metabolized in the liver to the mutagenic agent acetaldehyde, which reaches the general circulation and is considered to be responsible for the carcinogenicity of alcohol. It is not clear, however, why alcohol use increases the risk of some cancers and not others $[1,2,6,7]$. The breast cancer risk can be explained by the increased levels of estrogens observed after alcohol consumption; estrogens play an important role in breast carcinogenesis [8]. However, since acetaldehyde is mostly produced in the liver, it is not clear why light alcohol consumption increases the risk of cancer of the oral cavity, pharynx and esophagus but not of the liver and other organs. Epidemiological studies have consistently shown that alcohol consumption causes a local carcinogenic effect. Researchers assessing differences in the carcinogenicity of alcohol on subsites of the head and neck have observed a high risk for anatomical sites in closest contact on ingestion of alcohol, such as the mobile part of the tongue and the hypopharynx [1]. Variations among tissues in the levels of isoenzymes involved in ethanol detoxification cannot explain the local carcinogenic effect of alcohol in individuals with functional isoenzymes [1]. Currently, the biological mechanism by which alcohol consumption imposes a high risk of cancer of the oral cavity, pharynx and esophagus remains to be elucidated.

\section{Discussion}

Exposure to mutagenic agents is an important cause of cancer; for example, tobacco use is a mutagenic factor that increases the risk of oral cavity cancer and pharyngeal cancer by about 5 to 10 times [9]. However, ageing and the self-renewal capacity of tissues increase the risk of cancer much more than any known mutagenic agent [10]. For example, the risk of being diagnosed with cancer of the oral cavity and pharynx is about 80 times higher for people over 60 years old than for people under 30 [11]. In addition, tissues with a high self-renewal capacity are known to give rise to cancer even a million times more often than tissues without this capacity [12]. Recent analyses of the stinking differences in cancer risk by age and among tissues indicate that the main biological cause of cancer is the accumulation of cell divisions in stem cells [12-16]. The marked increase in cancer incidence with age observed for most cancers [11] strongly suggests that cancer originates in stem cells (Figure 1) [14,15]. The striking correlation between the risk of cancer in a tissue and the lifetime number of stem cell divisions occurring in that tissue (Spearman's rho $=0.81 ; P<3.5 \times 10^{-8}$ ) [12] indicates that the main biological cause of cancer is the accumulation of cell divisions in stem cells [14-17]. Ageing and the self-renewal capacity of tissues increase the risk of cancer because they induce the accumulation of cell divisions in stem cells. Every time a cell divides, it becomes exposed to unavoidable mistakes associated with cell division (e.g., mutations arising during DNA replication and chromosomal aberrations occurring during mitosis) and also becomes highly vulnerable to endogenous and exogenous mutagens (e.g., reactive oxygen species, tobacco 
carcinogens and acetaldehyde). Therefore, the more cell divisions a stem cell accumulates over a lifetime, the more DNA alterations it will accumulate, and the higher will be its risk of malignant transformation. This implies that cancer is not only caused by mutagenic agents, but also by any factor that promotes the accumulation of cell divisions in stem cells. Cell death occurring during physiological tissue self-renewal, or after exposure to cytotoxic agents, is a critical trigger for the division of stem cells. Any factor that causes cell death will promote the accumulation of cell divisions in stem cells and, therefore, their risk of malignant transformation [14-17].

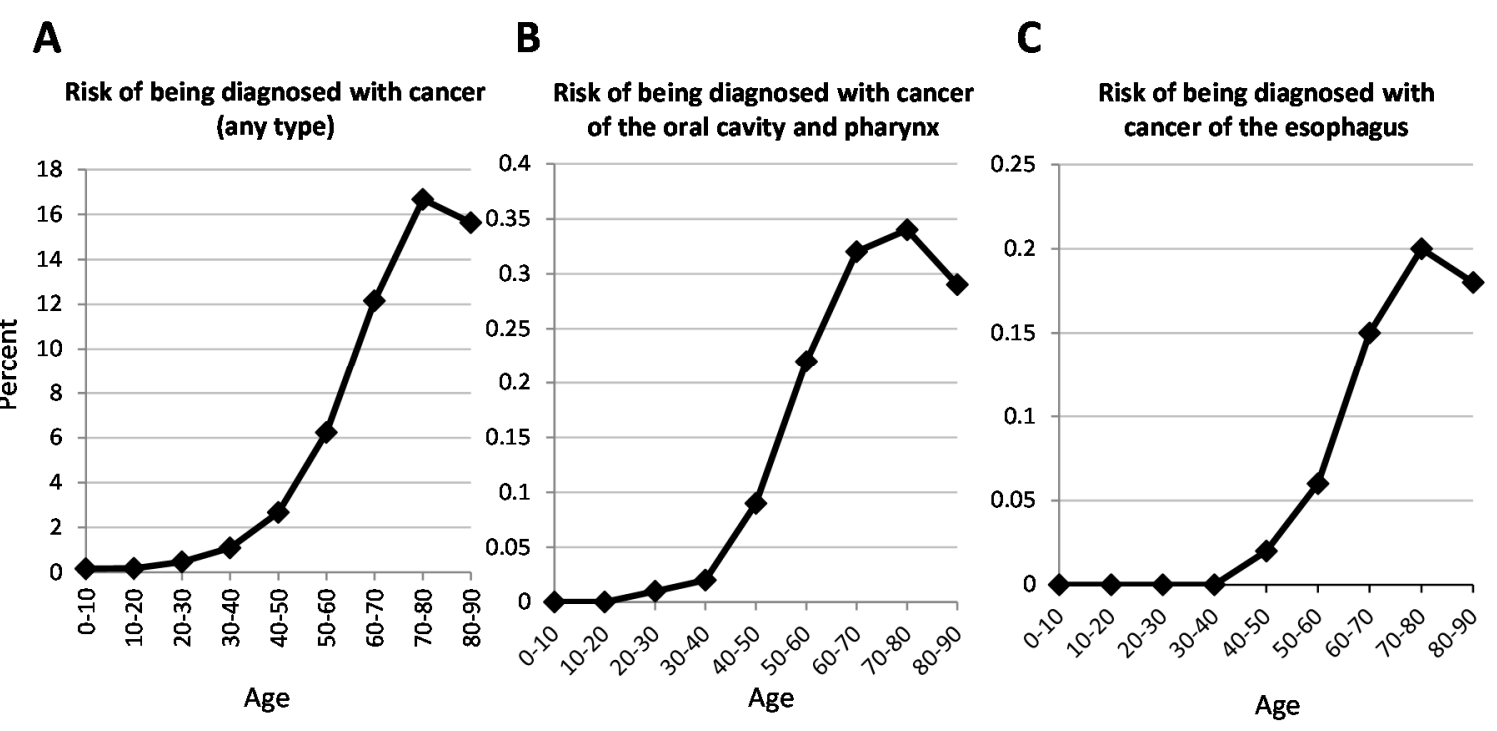

D

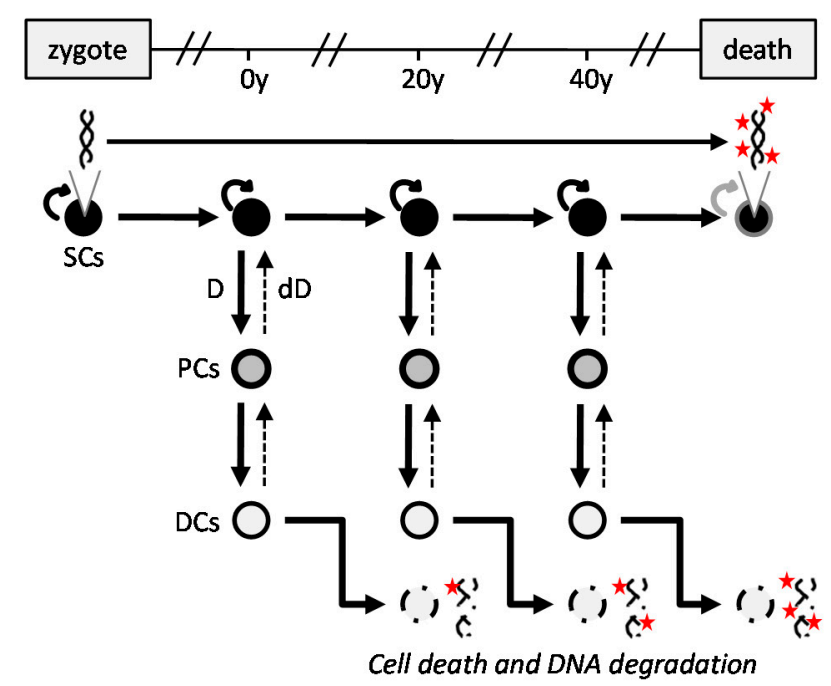

Figure 1. Cancer risk by age indicates that cancer originates in stem cells. Like for most cancers (A), the risk of being diagnosed with cancer of the oral cavity and pharynx (B) and esophagus (C) increases dramatically with age [11]. This implies that most cancers are the end-result of several successive cellular changes; if cancer were caused by only one cellular change, this change could occur at any moment in life and cancer incidence would be rather similar at different ages [13]. Importantly, DNA is the only cellular component that can accumulate changes throughout life, and stem cells are the only cells that can keep our DNA from the beginning of life (zygote) until death (D). At different moments in life, stem cells pass a copy of our DNA to progenitor cells (transit amplifying cells), which in turn pass it to the differentiated cells that form the bulk of the tissues. 
However, these DNA copies are degraded when differentiated cells die to be replaced by new cells. As we progress from zygote to death, our stem cells acquire DNA changes that increase our risk of cancer. If cancer originated in non-stem cells, progenitor cells or differentiated cells created at age 0 , 20 or 40 would have the same probability of acquiring the first cancer-related change. After a period of e.g. 30 years (during which the cell would acquire the rest of the cancer-related changes required for carcinogenesis), cancer incidence would be rather similar at ages 30, 50 or 70 . This is incompatible with cancer statistics shown in panels A, B and C. Therefore, the marked increase in cancer incidence with age indicates that most, if not all, cancers originate and largely develop in stem cells. The known decline in stem cell functionality with age occurring in most tissues [26] can explain why cancer risk decreases late in life. See reference [16] for additional details. Curved arrows represent self-renewal capacity. Broken arrows represent less-favored pathways. Stars represent DNA alterations. SCs: stem cells; PCs: progenitor cells; DCs: differentiated cells; D: differentiation; dD: dedifferentiation. Figure adapted from reference [16].

Importantly, ethanol causes cell death at the concentrations present in alcoholic beverages [18-21]. This effect is well known by Pharmacologists using ethanol as a drug solvent in cell culture experiments. The cytotoxicity of ethanol depends both on the exposure time and on its concentration. Most cells die after a one-hour exposure to 5-10\% ethanol or after a 15-second exposure to $30-40 \%$ ethanol [18]. Because dead cells cannot give rise to cancer, the known cytotoxic activity of ethanol at concentrations present in alcoholic beverages has probably been discarded as a possible mechanism by which alcohol consumption increases the risk of developing the disease.

Alcohol consumption probably causes a local cytotoxic effect on the cells lining the epithelial tissues of the oral cavity, pharynx and esophagus. The stem cells located in deeper layers of the mucosa, which are not directly exposed to ethanol, must divide to replace the dead cells. When they divide, they become exposed to unavoidable and carcinogen-mediated DNA alterations that increase their risk of malignant transformation. It has been estimated that the stem cells in the oral mucosa divide every 14 to 20 days and those of the esophagus every 21 days [12]. These division rates probably increase when cytotoxic concentrations of ethanol are ingested regularly. Alcohol consumption may therefore increase the risk of developing cancer in the oral cavity, pharynx and esophagus by promoting the accumulation of cell divisions in the stem cells that maintain these tissues in homeostasis.

Because the cytotoxic effect of ethanol is concentration-dependent [18], the risk of these cancers can increase not only with increasing amounts of ethanol, but also with increasing ethanol concentrations in the ingested drinks. In other words, an ounce of whisky is probably more carcinogenic when taken undiluted than when taken diluted. The local cytotoxic activity of ethanol may therefore explain why alcohol consumption increases the risk of oral cavity, pharyngeal and esophageal cancers even in people ingesting low amounts of ethanol (light drinkers). Ethanol is diluted by the stomach content to concentrations that no longer cause cell death; the local carcinogenic effect of ethanol is therefore attenuated when alcoholic drinks reach a non-empty stomach. 
There is a known synergistic effect of alcohol and tobacco consumption on the risk of cancer of the oral cavity, pharynx and esophagus [22-24]. For example, the risk of oral cavity and pharynx cancers is about 5-10 times higher for heavy smokers than for nonsmokers, and about 5 times higher for heavy drinkers than for nondrinkers. In people who are both heavy smokers and heavy drinkers, the risk of these cancers is about 35 times higher than for people who neither smoke nor drink [22]. It is well known that the DNA of dividing cells is more vulnerable to mutagenic agents than the DNA of non-dividing cells. During cellular quiescence, the DNA is less accessible to genotoxic agents because it is highly packaged into chromatin and because it is also protected by the nuclear membrane. When the cell divides, the DNA unwinds to be copied during DNA replication and the nuclear envelope disappears during mitosis; these cellular events facilitate the interaction of DNA-damaging agents with the DNA of the cell. Dividing cells are therefore more vulnerable than quiescent cells to the genotoxic activity of DNA-damaging agents. The local cytotoxic activity of ethanol may explain the synergistic effect of alcohol and tobacco consumption on the risk of cancer of the oral cavity, pharynx and esophagus. As explained previously, the cytotoxic effect of ethanol on the cells lining these tissues activates the division of the stem cells located in deeper layers to replace the dead cells. By activating their division, alcohol consumption leaves the DNA of stem cells highly exposed to the DNA-damaging activity of tobacco carcinogens. The fact that this synergistic effect only occurs in tissues in closest contact with alcohol supports this possible explanation.

In summary, the cytotoxic activity of ethanol at the concentrations present in alcoholic beverages can explain why alcohol consumption preferentially exerts a local carcinogenic effect. Understanding the biological mechanisms by which alcohol causes cancer is important to reinforce the epidemiological evidence and to raise public awareness of the strong link between alcohol consumption and the development of the disease [2,25].

\section{Conflict of interests}

None.

\section{References}

1. International Agency for Research on Cancer. Alcohol drinking. IARC monographs on the evaluation of carcinogenic risks to humans, vol 44. Lyon: IARC, 1988. Updated in Volume 100E (2012): http://monographs.iarc.fr/ENG/Monographs/vol100E/mono100E-11.pdf

2. Connor J. Alcohol consumption as a cause of cancer. Addiction 2016; doi: 10.1111/add.13477

3. Bagnardi V., Rota M., Botteri E., Tramacere I., Islami F., Fedirko V. et al. Alcohol consumption and site-specific cancer risk: a comprehensive dose-response meta-analysis. $\mathrm{Br}$ J Cancer 2015; 112: 580-93.

4. Bagnardi V., Rota M., Botteri E., Tramacere I., Islami F., Fedirko V. et al. Light alcohol drinking and cancer: a meta-analysis. Ann Oncol 2013; 24: 301-8. 
5. Praud D., Rota M., Rehm J., Shield K., Zatonski W., Hashibe M. et al. Cancer incidence and mortality attributable to alcohol consumption. Int J Cancer 2016; 138: 1380-7.

6. Boffetta P., Hashibe M. Alcohol and cancer. Lancet Oncol 2006; 7: 149-56.

7. Seitz H. K., Stickel F. Molecular mechanisms of alcohol-mediated carcinogenesis. Nat Rev Cancer 2007; 7: 599-612.

8. Dorgan J. F., Baer D. J., Albert P. S., Judd J. T., Brown E. D., Corle D. K. et al. Serum hormones and the alcohol-breast cancer association in postmenopausal women. J Natl Cancer Inst 2001; 93: 710-5.

9. Tobacco smoke and involuntary smoking IARC Monogr Eval Carcinog Risks Hum 2004; 83:11438: 1-1438.

10. Lopez-Lazaro M. What Is the Main Cause of Cancer? Preprints 2016, 2016080130; doi: 10.20944/preprints201608.0130.v1

11. SEER Cancer Statistis Review, 1975-2012. 2015. http://seer.cancer.gov/archive/csr/1975_2012/results_merged/topic_lifetime_risk.pdf

12. Tomasetti C., Vogelstein B. Cancer etiology. Variation in cancer risk among tissues can be explained by the number of stem cell divisions. Science 2015; 347: 78-81.

13. Armitage P., Doll R. The age distribution of cancer and a multi-stage theory of carcinogenesis. Br J Cancer 1954; 8: 1-12.

14. Lopez-Lazaro M. Stem cell division theory of cancer. Cell Cycle 2015; 14: 2547-8.

15. Lopez-Lazaro M. Cancer arises from stem cells: opportunities for anticancer drug discovery. Drug Discov Today 2015; 20: 1285-7.

16. Lopez-Lazaro M. Understanding cancer: 15 questions and answers. ResearchGate, 2016; DOI: 10.13140/RG.2.1.4180.6323: http://dx.doi.org/10.13140/RG.2.1.4180.6323

17. Lopez-Lazaro M. Understanding why aspirin prevents cancer and why consuming very hot beverages and foods increases esophageal cancer risk. Controlling the division rates of stem cells is an important strategy to prevent cancer. Oncoscience 2015; 2: 849-56.

18. Tapani E., Taavitsainen M., Lindros K., Vehmas T., Lehtonen E. Toxicity of ethanol in low concentrations. Experimental evaluation in cell culture. Acta Radiol 1996; 37: 923-6.

19. Le T. D., Do T. A., Yu R., Yoo H. Ethanol elicits inhibitory effect on the growth and proliferation of tongue carcinoma cells by inducing cell cycle arrest. Korean J Physiol Pharmacol 2012; 16: 153-8.

20. Slomiany B. L., Piotrowski J., Piotrowski E., Slomiany A. Induction of buccal mucosal apoptosis with chronic alcohol ingestion. Biochem Mol Biol Int 1998; 44: 381-9.

21. Slomiany B. L., Piotrowski J., Slomiany A. Chronic alcohol ingestion enhances tumor necrosis factor-alpha expression and salivary gland apoptosis. Alcohol Clin Exp Res 1997; 21: 1530-3.

22. Blot W. J., McLaughlin J. K., Winn D. M., Austin D. F., Greenberg R. S., Preston-Martin S. et al. Smoking and drinking in relation to oral and pharyngeal cancer. Cancer Res 1988; 48: 3282-7. 
23. Hashibe M., Brennan P., Chuang S. C., Boccia S., Castellsague X., Chen C. et al. Interaction between tobacco and alcohol use and the risk of head and neck cancer: pooled analysis in the International Head and Neck Cancer Epidemiology Consortium. Cancer Epidemiol Biomarkers Prev 2009; 18: 541-50.

24. Prabhu A., Obi K. O., Rubenstein J. H. The synergistic effects of alcohol and tobacco consumption on the risk of esophageal squamous cell carcinoma: a meta-analysis. Am J Gastroenterol 2014; 109: 822-7.

25. Burki T. K. Low public awareness of link between cancer and alcohol. Lancet Oncol 2016; 17: e184-2045.

26. Goodell M. A., Rando T. A. Stem cells and healthy aging. Science 2015; 350: 1199-204.

(C) 2016 by the author; licensee Preprints, Basel, Switzerland. This article is an open access article distributed under the terms and conditions of the Creative Commons by Attribution (CC-BY) license (http://creativecommons.org/licenses/by/4.0/). 